\title{
ASYMPTOTIC SOLUTIONS FOR UNSTEADY FLOW IN CASCADES*
}

\author{
BY \\ ODDVAR O. BENDIKSEN \\ Princeton University
}

\begin{abstract}
Summary. Asymptotic methods are presented for obtaining approximate solutions to unsteady flows in oscillating cascades. The formulation is in the framework of linearized potential flow, and the problems considered include low-frequency and low-solidity expansions for subsonic and supersonic cascades. For the supersonic cascade, simple formulas are obtained for the unsteady lift and moment, valid to first order in a frequency parameter. It is shown that terms of successive orders can be obtained by solving a sequence of quasi-static problems, with the effective upwash at each step modified by the lower-order solutions. The approach is in the spirit of matched asymptotic expansions, and different expansions based on different limit processes are sought for the subresonant and superresonant regions. For cascades in subsonic axial flow, the acoustic resonance phenomenon leads to a nonuniformity with respect to the interblade phase angle. The location of the singularity can be moved by suitably redefining the limit process, permitting uniformly valid expansions to be obtained separately for the subresonant and superresonant regions. Numerical comparisons with the full unsteady solution indicate that the present approximations are remarkably accurate in the range of reduced frequencies of interest in aeroelastic analyses.
\end{abstract}

1. Introduction. For reasons which are not clear, asymptotic solutions to unsteady cascade problems have not been explored to the same degree as in the corresponding isolated wing problems. A possible explanation is that analytical difficulties and uncertainties about the validity of any approximations thus obtained have discouraged such efforts. This paper is an attempt to illustrate how the methods of asymptotic analysis can be applied to obtain useful engineering approximations and also an improved understanding of the problems themselves.

The unsteady supersonic cascade is probably the most studied problem in this respect. The motivation for many of these investigations is no doubt the increased recognition of supersonic flutter as a serious and often limiting design constraint for advanced fan rotors. For current technology rotors, it is necessary to consider the subsonic leading edge locus

\footnotetext{
${ }^{*}$ Received May 7, 1985.
} 
problem, where the velocity component normal to the leading edge is subsonic. Kurosaka [1] appears to have obtained the first asymptotic solution to this problem, valid to first order in a reduced frequency parameter. Similar low-frequency expansions have since been obtained by a number of investigators [2-4]. A characteristic feature of these solutions is a singularity at an interblade phase angle of $\sigma=0$, indicating a breakdown of the solutions as $\sigma \rightarrow 0$ or $2 \pi$. The origin of this nonuniformity is now well understood; it arises from the collapse of the superresonant region to the point $k=\sigma=0$ as the reduced frequency $k$ approaches zero. Since the expansions are not valid in the superresonant region or at the resonance points, a singularity at $\sigma=0$ is to be expected.

These observations cast doubt on the practical usefulness of approximation of this type in aeroelastic studies. In a recent study [5], however, a uniformly valid solution was obtained for the subresonant region by suitably redefining the underlying limit process $k \rightarrow 0$. In the present paper it is demonstrated how different expansions, based on different limit processes, can be constructed for the subresonant and superresonant regions, thus covering the entire range of interblade phase angles. These ideas are then shown to be extendable to subsonic flows. Also treated is the low-solidity cascade, where asymptotic expansions are obtained in terms of a blade spacing parameter. Such expansions provide insight into the importance of cascade effects in low-solidity rotors, such as propellers and propfans.

2. Governing equations. Within the framework of linearized potential flow, through a harmonically oscillating cascade, the spatial dependence of the perturbation quantities satisfy the Helmholtz equation. The associated boundary value problem for the infinite two-dimensional cascade can be recast in the form of a set of dual integral equations involving the upwash $\bar{v}_{0}(\bar{x})$ and the pressure loading $\left[\bar{p}_{0}(\bar{x})\right]$ on the zeroth (reference) blade and its extension $-\infty<\bar{x}<\infty ; \bar{y}=0$ :

$$
\begin{gathered}
\bar{v}_{0}(\bar{x})=\frac{i \beta}{2} e^{ \pm i \bar{k} M \bar{x}} \int_{-\infty}^{\infty} G(\tau) F_{0}(\tau) e^{i \bar{x} \tau} d \tau, \\
{\left[\bar{p}_{0}(\bar{x})\right]=e^{ \pm i \bar{k} M \bar{x}} \int_{-\infty}^{\infty} F_{0}(\tau) e^{i \bar{x} \tau} d \tau,}
\end{gathered}
$$

where the plus sign in the exponents is to be taken in the subsonic case and the minus sign in the supersonic case. The derivation of these equations is given in $[6,7]$ for the subsonic case and in $[4,8]$ for the supersonic case. Here $F_{0}$ is an unknown function to be determined, and the reduced kernel $G(\tau)$ is

$$
G(\tau)= \begin{cases}\frac{\sqrt{\bar{k}^{2}-\tau^{2}} \sin \left(\bar{s}_{2} \beta \sqrt{\bar{k}^{2}-\tau^{2}}\right)}{(\tau+\bar{k} / M)\left[\cos \left(\bar{s}_{2} \beta \sqrt{\bar{k}^{2}-\tau^{2}}\right)+\cos \left(\Omega-\bar{s}_{1} \tau\right)\right]} & (\text { for } M<1), \\ \frac{\sqrt{\tau^{2}-\bar{k}^{2}} \sin \left(\bar{s}_{2} \beta \sqrt{\tau^{2}-\bar{k}^{2}}\right)}{(\tau-\bar{k} / M)\left[\cos \left(\bar{s}_{2} \beta \sqrt{\tau^{2}-\bar{k}^{2}}\right)-\cos \left(\Omega-\bar{s}_{1} \tau\right)\right]} & (\text { for } M>1) .\end{cases}
$$

The notation is the same as in [8]: $k=\omega b / U, \bar{x}=x / b, b=$ semichord, $\beta^{2}=M^{2}-1$ (supersonic) or $1-M^{2}$ (subsonic), $M=$ Mach no., $\bar{k}=k M / \beta^{2}, \Omega=\sigma+\bar{s}_{1} \bar{k} M$ (supersonic) or $\sigma-\bar{s}_{1} \bar{k} M$ (subsonic), $\bar{s}_{1}=\bar{s} \sin \theta, \bar{s}_{2}=\bar{s} \cos \theta, \bar{s}=s / b, s=$ blade spacing 
along leading edge locus, and $\theta=$ stagger angle. Unless otherwise noted, an overbar signifies a nondimensional quantity: lengths with respect to semichord $b$, velocities with respect to flow velocity $U$ at infinity, and pressures with respect to $\rho_{0} U^{2}$.

To ensure the existence of certain integrals and the convergence of the infinite series representation of $G(\tau)$, the reduced frequency $k$ is assumed to have a small negative imaginary part; $k=k_{1}-i k_{2}, 0<k_{2} \ll 1$, and the limit $k_{2} \rightarrow 0^{+}$is implied throughout. Also in Eq. (1) and all subsequent Cauchy-type integrals, the Cauchy principal value is implied. The radiation condition and boundedness at infinity require different integration paths and branch cut choices for the square root in the subsonic and the supersonic case, as illustrated in Fig. 1. In the subsonic case the integration path remains fixed along the
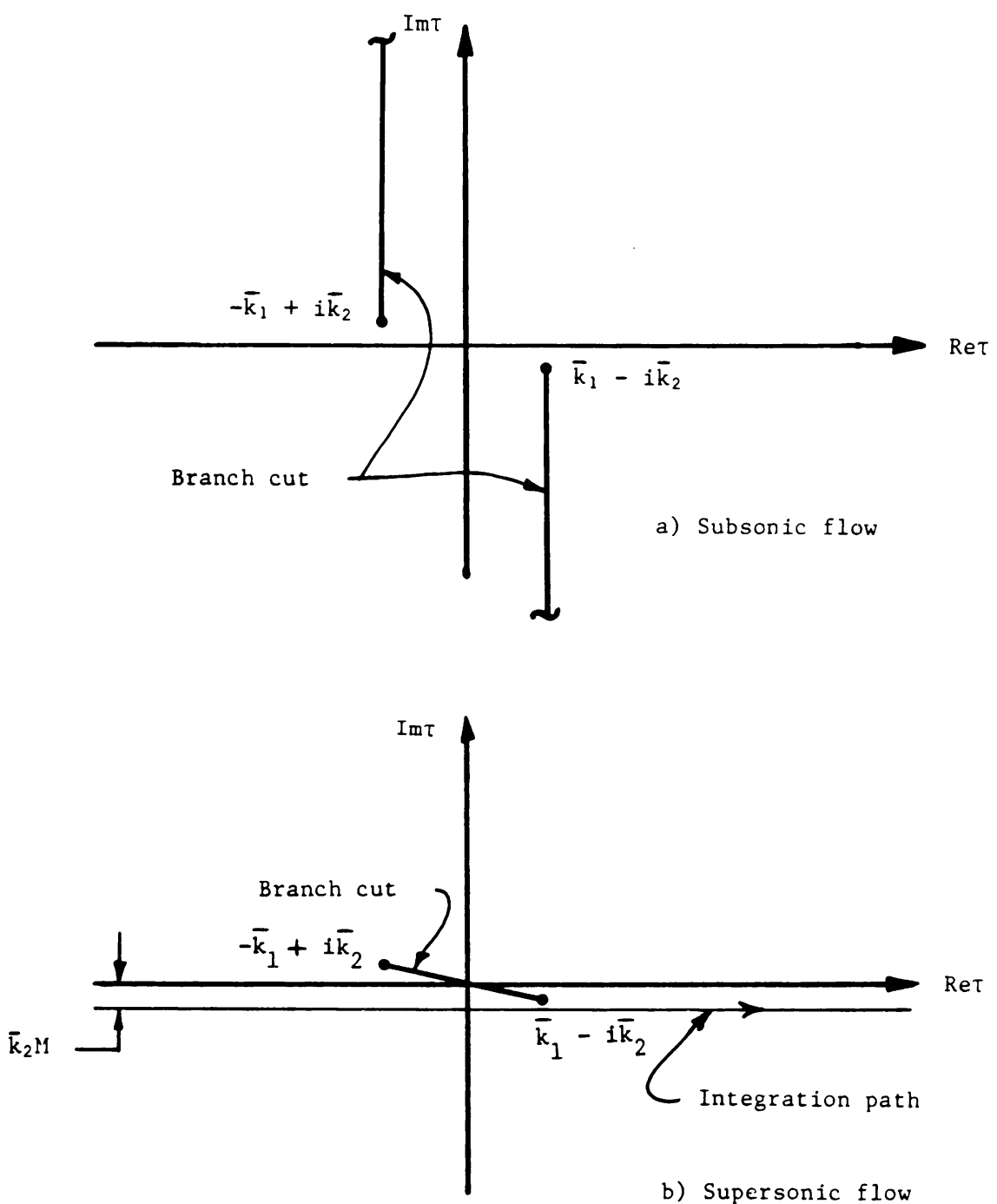

Fig. 1. Branch cuts for the square root in the cascade kernel and integration path for Eqs. $(1,2)$. 
real $\tau$-axis, while in the supersonic case the path is displaced by an amount $-\bar{k}_{2} M$, approaching the real axis from below in the limit $k_{2} \rightarrow 0^{+}$. Incidentally, the branch point singularities of $G$ are removable, but it is sometimes convenient to split up $G$ in a manner which reintroduces the branch point singularities. They also occur in the isolated airfoil kernels.

The upwash is known for $|\bar{x}|<1$ from the flow tangency conditions, and pressure continuity across the wakes and ahead of the cascade requires the jump $\left[\bar{p}_{0}(\bar{x})\right]$ to vanish for $|\bar{x}|>1$. For blades undergoing bending and torsional oscillations with amplitudes $\bar{h}_{0}$ and $\alpha_{0}$, referenced to an elastic axis located a distance $\bar{x}=a$ behind the mid-chord, the boundary conditions become

$$
\begin{gathered}
\bar{v}_{0}(\bar{x})=-i k \bar{h}_{0}-[1+i k(\bar{x}-a)] \alpha_{0} \text { for }|\bar{x}|<1, \\
{\left[\bar{p}_{0}(\bar{x})\right]=0 \text { for }|\bar{x}|>1 .}
\end{gathered}
$$

Thus, the solution of the problem has been reduced to the determination of the unknown function $F_{0}(\tau)$. Similar dual integral equation formulations of a number of mixed boundary value problems can be found in the book by Sneddon [9]. In view of Eq. (2), $F_{0}(\tau \pm \bar{k} M)$ is simply the Fourier transform of the blade loading. One convenient feature of this formulation is that the lift and moment (about mid-chord) amplitudes are directly related to $F_{0}(\tau)$ and its derivative at the single point $\tau=\tau_{0}$ :

$$
\begin{gathered}
L_{0}=-2 \pi \rho_{0} U^{2} b F_{0}\left(\tau_{0}\right), \\
M_{0_{\mathrm{mc}}}=2 \pi i \rho_{0} U^{2} b^{2} F_{0}^{\prime}\left(\tau_{0}\right),
\end{gathered}
$$

positive up and clockwise, respectively, and where $\tau_{0}=-\bar{k} M$ in subsonic flow and $\tau_{0}=+\bar{k} M$ in supersonic flow.

3. Low-frequency expansions. In constructing low-frequency approximations for the solution of Eqs. $(1,2)$, the singularities of $G(\tau)$ play a major role. It is therefore necessary to distinguish between operating regions where these singularities differ in type or number, i.e., subsonic vs. supersonic flow and subresonant vs. superresonant operation. The approach is in the spirit of limit process expansions [10], and it is important to keep in mind what variables are considered fixed in the associated limit $k \rightarrow 0$.

Supersonic Flow. Let $k=\varepsilon \ll 1$, and assume that an asymptotic expansion for the solution $F_{0}$ is of the form

$$
F_{0}(\tau+\bar{k} M)=f_{0}(\tau)+\nu_{1}(\varepsilon) f_{1}(\tau)+\nu_{2}(\varepsilon) f_{2}(\tau)+\cdots,
$$

where $\nu_{n+1} / \nu_{n} \rightarrow 0$ as $\varepsilon \rightarrow 0$. Asymptotic here means that the error can be made arbitrarily small, with a finite number of terms, by making $\varepsilon$ sufficiently small. Since the small parameter $\varepsilon=k$ enters the boundary conditions, Eq. (4), we write

$$
\begin{gathered}
\bar{v}_{0}(\bar{x})=\bar{v}_{0}^{(0)}(\bar{x})+\varepsilon \bar{v}_{0}^{(1)}(\bar{x})+\cdots, \\
{\left[\bar{p}_{0}(\bar{x})\right]=\left[\bar{p}_{0}^{(0)}(\bar{x})\right]+\nu_{1}(\varepsilon)\left[\bar{p}_{0}^{(1)}(\bar{x})\right]+\cdots .}
\end{gathered}
$$

For bending-torsion oscillations of the type considered here, $\bar{v}_{0}^{(0)}=\bar{v}_{0_{b}}-\alpha_{0}$ and $\bar{v}_{0}^{(1)}=$ $i(a-\bar{x}) \alpha_{0}$. Note that at this point the bending velocity amplitude $\bar{v}_{0_{b}}\left(=-i k \bar{h}_{0}\right)$ has been assumed $O(1)$ rather than $O(\varepsilon)$. 
When Eqs. $(6,7)$ are substituted into Eqs. $(1,2)$ and the limits $\varepsilon \rightarrow 0$ repeatedly applied, a series of dual integral equations are obtained for the $f_{n}(\tau)$ 's, $n=0,1,2, \ldots$ :

$$
\begin{gathered}
\bar{v}_{0}^{(n)}(\bar{x})=\frac{i \beta}{2} \int_{-\infty}^{\infty} G_{\infty}\left(\tau-i 0^{+}\right) f_{n}(\tau) e^{i \bar{x} \tau} d \tau+\Delta \bar{v}_{0}^{(n)}(\bar{x}), \\
{\left[\bar{p}_{0}^{(n)}(\bar{x})\right]=\int_{-\infty}^{\infty} f_{n}(\tau) e^{i \bar{x} \tau} d \tau=0 \quad \text { for }|\bar{x}|>1,}
\end{gathered}
$$

where

$$
\begin{aligned}
& G_{\infty}(\tau)=\frac{\sin \left(\bar{s}_{2} \beta \tau\right)}{\cos \left(\bar{s}_{2} \beta \tau\right)-\cos \left(\Omega-\bar{s}_{1} \tau\right),} \\
& \Delta \bar{v}_{0}^{(n)}(\bar{x})=\frac{i \beta}{2} \lim _{\varepsilon \rightarrow 0}\left\{\frac { 1 } { \nu _ { n } ( \varepsilon ) } \int _ { - \infty } ^ { \infty } \left[G\left(\tau+\varepsilon M^{2} / \beta^{2} ; \varepsilon\right) F_{0}\left(\tau+\varepsilon M^{2} / \beta^{2}\right)\right.\right. \\
& \left.\left.-G_{\infty}\left(\tau+\varepsilon M^{2} / \beta^{2}\right) \sum_{m=0}^{n} \nu_{m}(\varepsilon) f_{m}(\tau)\right] e^{i \bar{x} \tau} d \tau\right\},
\end{aligned}
$$

where $\varepsilon=k=k_{1}-i k_{2}, k_{2} \ll 1$, and $k_{2} \rightarrow 0^{+}$as $\varepsilon \rightarrow 0$. $G_{\infty}$, Eq. (10), is the asymptotic form of $G$ for $k=0$ with $\Omega$ fixed, or as $|\tau| \rightarrow \infty$. Thus $G_{\infty}(\tau)$ is the reduced kernel associated with the quasi-static problem, or the steady problem if $\Omega=0$, for which an exact solution has been obtained [4]. This solution can be used to solve Eqs. $(8,9)$ by treating $\left(\bar{v}_{0}^{(n)}-\Delta \bar{v}_{0}^{(n)}\right)$ as the equivalent quasi-static upwash at each step. As long as $\sigma$ is not too close to one of the resonance points, the term $\Delta \bar{v}_{0}^{(n)}$ can be shown to depend only on the solutions of lower order and is therefore known at each step. The solution for the $n$th correction term is then

$$
f_{n}(\tau)=\sum_{m=0}^{\infty} a_{m}^{(n)}\left\{j_{m}(\tau)+\frac{i^{m}}{2} \sum_{r=1}^{N_{r}} R_{r} Q_{m}^{r}(\tau)\right\},
$$

where

$$
a_{m}^{(n)}=(-i)^{m} \frac{(2 m+1)}{\pi \beta} \int_{-1}^{1}\left[\bar{v}_{0}^{(n)}(x)-\Delta \bar{v}_{0}^{(n)}(\bar{x})\right] P_{m}(\bar{x}) d \bar{x},
$$

$j_{m}(\tau)$ is the spherical Bessel function of the first kind of order $m$, and $P_{m}(\bar{x})$ is the Legendre polynomial of degree $m$. The last sum in Eq. (12a) is due to Mach wave reflections, if present, and expressions for $R_{r}$ and $Q_{m}^{r}$ can be found in [4].

Thus the asymptotic expansions for $F_{0}$, Eq. (6), can in principle be calculated to successive orders $1, \nu_{1}, \nu_{2}, \ldots$ by solving a series of quasi-static problems. The effective upwash at each step is modified by the lower-order solutions through $\Delta \bar{v}_{0}^{(n)}$. A similar approach, also involving the solution of a sequence of simplified integral equations, has been used by Graham and Kullar [11] to obtain expansions for an isolated airfoil in unsteady flow. As they point out, this process is equivalent to the use of matched asymptotic expansions, but with the matching replaced by the successive dependence of the integral equations on the solutions of lower order.

(a) Subresonant region. As long as the interblade phase angle is not too close to the resonance points, the zeroth-order term $f_{0}$ in the expansion for $F_{0}$ coincides with the quasi-static solution. The next term is then of order $\nu_{1}=\varepsilon$, and the solution complete to 
first order in $\varepsilon=k$ is

$$
\begin{gathered}
a_{0}=\frac{2 \bar{v}_{0_{b}}}{\pi \beta}\left[1-\frac{i k}{\beta^{2}}\left\{1-2 S^{*}\left(\Omega^{*}\right)\right\}\right]-\frac{2 \alpha_{0}}{\pi \beta}\left[1-\frac{i k}{\beta^{2}}\left\{1+a \beta^{2}-2 S^{*}\left(\Omega^{*}\right)\right\}\right], \\
a_{1}=-\frac{2 k}{\pi \beta^{3}}\left[\bar{v}_{0_{b}}+\left(\beta^{2}-1\right) \alpha_{0}\right], \quad a_{m}=0, \quad m=2,3,4, \ldots,
\end{gathered}
$$

in agreement with Eqs. $(16,17)$ of [5]. Here $a_{m}=a_{m}^{(0)}+\varepsilon a_{m}^{(1)}+\cdots$, and $S^{*}$ is a function arising from the upwash term $\bar{v}_{0}^{(1)}$ defined by

$$
S^{*}=\left\{\begin{array}{ll}
S \kappa_{2} / 2 & \text { for } 0<\kappa_{2}<2, \\
S & \text { for } \kappa_{2}>2
\end{array} \quad S\left(\Omega^{*}\right)=\frac{1}{2}+\frac{i}{2} \cot \frac{\Omega^{*}}{2},\right.
$$

with $\Omega^{*}=\Omega-\kappa_{2} \bar{k} / M=\sigma+k\left(\bar{s}_{1}+\bar{s}_{2} / \beta\right)$ and $\kappa_{2}=\bar{s}_{1}-\beta \bar{s}_{2}$. Physically, terms involving $S^{*}$ in $\Delta \bar{v}_{0}^{(1)}$ represent corrections to the $O(k)$ quasi-static upwash resulting from upward-propagating disturbances from the blades and vortex wakes below the reference blade. The fact that $S^{*}$ blows up as $\Omega^{*} \rightarrow 2 \pi \nu ; \nu=0, \pm 1, \pm 2, \ldots$ is connected with the resonance phenomenon predicted by most linearized cascade theories. Only one of these singularities lies in the range $0 \leqslant \sigma<2 \pi$, and this pole can be shown to lie inside the superresonant region.

A suitable limit process for the subresonant region is to keep $\Omega^{*}$ (and all other variables except $\sigma$ ) fixed as $k \rightarrow 0$ [5]. This preserves the locations of the singularities of $S$ and permits a uniformly valid solution to be obtained, to $O(k)$ :

$$
\begin{gathered}
a_{0}=\frac{2\left[\bar{v}_{0_{b}}-\alpha_{0}\{1-i a k\}\right]}{\pi \beta\left[1+i\left\{1-2 S^{*}\left(\Omega^{*}\right)-i T\right\} k / \beta^{2}\right]}, \\
a_{1}=-\frac{k}{\beta^{2}} a_{0}\left[1+2 i k S^{*}\left(\Omega^{*}\right)\right]-\frac{2 k \alpha_{0}}{\pi \beta},
\end{gathered}
$$

where

$$
T=\beta \sinh \left(\bar{s}_{2} k\right) /\left[\cosh \left(\bar{s}_{2} k\right)-\cos \Omega^{*}\right] .
$$

This solution agrees with [5] except for the term involving $T$. Although strictly speaking a second-order term for $\Omega^{*}$ fixed, it has been found to improve the accuracy of the approximations sufficiently to warrant inclusion. The differences between Eqs. (13) and (15) arise from the fact that close to the resonance points $S \sim O(1 / \varepsilon)$ as $\varepsilon \rightarrow 0$ with $\Omega^{*}$ fixed; therefore the term $\varepsilon \Delta \bar{v}_{0}^{(1)}$ grows to order one and must be included in the zeroth-order equation to preserve the uniformity of the expansion. This makes the upwash correction term $\varepsilon \Delta \bar{v}_{0}^{(1)}$ also a function of $a_{0}^{(0)}$ in Eq. (12b) and leads to the solution in Eq. (15).

(b) Superresonant region. Formally, the expansion procedure for the superresonant region is the same as for the subresonant region, and Eqs. (6-12) apply. However, it is clear that in the superresonant case one cannot keep $\Omega^{*}$ fixed as $k \rightarrow 0$, since this results in a nonuniformity within the superresonant region. 
Mathematically, the difference between the two regions is the appearance, in the superresonant case, of one or more sets of complex poles in the kernel $G$, Eq. (3). The singularities of $G$ consist of a pole at $\tau=\bar{k} / M$ and an infinite set of poles at the points $\tau_{\nu}^{ \pm}$given by

$$
\bar{d}^{2} \tau_{\nu}^{ \pm}=-\bar{s}_{1}(2 \pi \nu-\Omega) \pm \beta \bar{s}_{2} \sqrt{(2 \pi \nu-\Omega)^{2}-(\bar{k} \bar{d})^{2}}, \quad \nu=0, \pm 1, \pm 2, \ldots
$$

where $\bar{d}^{2}=\bar{s}_{1}^{2}-\beta^{2} \bar{s}_{2}^{2}$. Recall that the reduced frequency $k$ has been assumed to have a small negative imaginary part, $-i k_{2}$, and the limit $k_{2} \rightarrow 0^{+}$is implied. This has the effect of displacing the "real" poles slightly from the integration path, so that in the limit $k_{2} \rightarrow 0^{+}$the set $\tau_{\nu}^{+}$approaches from above and the set $\tau_{\nu}^{-}$from below.

The case where the radical vanishes corresponds to the resonance condition, which from Eq. (17) implies that a pole of order two would occur for $k_{2}=0$. In the subresonant region, the radicand is always positive and all the poles $\tau_{\nu}{ }^{ \pm}$are real, corresponding to propagating acoustic waves. In the superresonant region, the radicand is negative for at least one value of $\nu$, and both spatially decaying and propagating waves are produced. The contribution from the pole at $\bar{k} / M$ represents the effect of shed vorticity from the cascade on the upwash on the reference blade.

To obtain useful expansions for the superresonant region one must therefore ensure that the contributions from the complex poles are evaluated correctly. For cascades of practical interest only one complex pair will occur in the low-frequency region presently considered, since $\bar{k} \bar{d}<2 \pi$ except under unusual circumstances. Let $\nu=\nu_{c}$ correspond to this pair. To capture its contribution to the integral in (11), consider the limit process with

$$
\eta=\left(2 \pi \nu_{c}-\Omega\right) / \bar{k}=\left[2 \pi \nu_{c}-\Omega(\varepsilon)\right] \frac{\beta^{2}}{\varepsilon M}
$$

fixed as $\varepsilon=k \rightarrow 0$. Keeping $\eta$ fixed is also equivalent to keeping $\left(2 \pi \nu_{c}-\Omega^{*}\right) / \bar{k}$ or $\left(2 \pi \nu_{c}-\sigma\right) / \bar{k}$ fixed; thus $\Omega, \Omega^{*}$, and $\sigma$ all approach $2 \pi \nu_{c}$ as $\varepsilon \rightarrow 0$. This preserves the residues of $G$ at the complex poles $\tau_{c}^{ \pm}=\tau_{\nu_{c}}{ }^{ \pm}$, which are given by

$$
G_{R c}=\frac{\beta \bar{s}_{2}\left[\left(\tau_{c}^{ \pm}\right)^{2}-\bar{k}^{2}\right]}{\left(\tau_{c}^{ \pm}-\bar{k} / M\right)\left[\bar{d}^{2} \tau_{c}^{ \pm}+\bar{s}_{1}(2 \pi \nu-\Omega)\right]},
$$

with $\nu=\nu_{c}$. Details of the calculation of the upwash correction term and the expansion coefficients can be found in [12]. For the case of no Mach wave reflections, the solution complete to order $\nu_{1}=\varepsilon=k$ is

$$
\begin{gathered}
a_{0}^{(0)}=\frac{2\left[\bar{v}_{0_{b}}-\alpha_{0}\right]}{\pi \beta\left[1-c_{0}\right]}, \quad a_{1}^{(0)} \simeq 0, \\
a_{0}^{(1)}=-4 i c_{3} a_{0}^{(0)} / 3\left(1-c_{0}\right), \quad a_{1}^{(1)}=-2 \alpha_{0} / \pi \beta-c_{5} a_{0}^{(0)},
\end{gathered}
$$


where

$$
\begin{aligned}
c_{0} & =1 / \kappa_{1}+1 / \kappa_{2}-G_{k}+2 i \operatorname{Im} G_{R c}, \\
c_{3} & =-b_{0}-i k\left(M \eta+\kappa_{2}\right) / 8 \beta^{4}, \\
c_{5} & =-b_{1}+1 / \beta^{2}, \\
b_{0} & =G_{k} / 2+d_{0}+\operatorname{Re}\left\{\mu_{c}^{+} G_{R c}\right\}, \\
b_{1} & =G_{k}-d_{1}+2 i \operatorname{Im}\left\{\mu_{c}^{+} G_{R c}\right\}, \\
d_{0} & =\left[1 / \kappa_{2}-1 / \kappa_{1}+\left(1 / \kappa_{2}^{2}-1 / \kappa_{1}^{2}\right) M \eta / \beta^{2}\right] / 2, \\
d_{1} & =1 / \kappa_{1}+1 / \kappa_{2}+\left(1 / \kappa_{1}^{2}+1 / \kappa_{2}^{2}\right) M \eta / \beta^{2}, \\
G_{k} & =2 \beta \bar{s}_{2} /\left[\beta^{2} \bar{s}_{2}^{2}+M^{2}\left(\eta+\bar{s}_{1} / M\right)^{2} / \beta^{2}\right], \\
\mu_{c}^{+} & =\left(\tau_{c}^{+}-\bar{k} M\right) / k, \quad \kappa_{1}=\bar{s}_{1}+\beta \bar{s}_{2}, \quad \kappa_{2}=\bar{s}_{1}-\beta \bar{s}_{2} .
\end{aligned}
$$

Subsonic Flow. The ideas used to construct asymptotic expansions for the supersonic case are readily extended to the subsonic case. By taking $F_{0}(\tau-\bar{k} M)$ in the form of Eq. (6) and proceeding as before, a sequence of quasi-static problems is again obtained. The resultant dual integral equations are of the same form as Eqs. $(8,9)$, but with $G_{\infty}$ now being the corresponding quasi-static kernel corresponding to the subsonic problem:

$$
G_{\infty}^{\mathrm{sub}}(\tau)=\frac{\sinh \left(\beta \bar{s}_{2} \tau\right)}{\cosh \left(\beta \bar{s}_{2} \tau\right)-\cos \left(\Omega-\bar{s}_{1} \tau\right)}
$$

where $\Omega=\sigma-\bar{s}_{1} \bar{k} M$, and the limit process must specify the dependence of $\Omega(\varepsilon)$ and $\sigma(\varepsilon)$ on $\varepsilon=k$ as $\varepsilon \rightarrow 0$. The choice of limit process must be based on an analysis of the behavior of the singularities of the subsonic kernel, Eq. (3), and the resultant effect on the upwash correction terms, Eq. (11) [in the subsonic case, the arguments $\tau+\varepsilon M^{2} / \beta^{2}$ in Eq. (11) become $\tau-\varepsilon M^{2} / \beta^{2}$ ].

Now the singularities of the subsonic and supersonic kernels are of the same type (simple poles), and all except for the "vorticity producing" pole at $\tau=-\bar{k} M(=+\bar{k} M$ for $M>1$ ) correspond to the generation of acoustic waves. The major difference between the subsonic and supersonic kernels is in the location of the "acoustic poles" $\tau_{\nu}^{ \pm}$in the complex plane. In the supersonic case these poles approach the integration path as $k_{2} \rightarrow 0$, corresponding to propagating waves, except in the superresonant region when one or more pairs of complex conjugate poles appear, representing spatially decaying waves. In the subsonic case, the situation is reversed. In the so-called subresonant region only attenuated waves are present $\left(\tau_{\nu}^{ \pm}\right.$poles are complex), while the superresonant region is characterized by the appearance of at least one pair $\tau_{\nu}{ }^{ \pm}$approaching the real $\tau$-axis.

Based on the work of Lane and Friedman [6] and Whitehead [7] for the full unsteady case, one would expect that the solution to the dual integral equations $(8,9)$ should be of 
the form

$$
f_{n}(\tau)=\sum_{m=0}^{\infty} a_{m}^{(n)} J_{m}(\tau)
$$

where $J_{m}(\tau)$ is the Bessel function of the first kind of order $m$. Such a series can be made to satisfy the Kutta condition at the blade trailing edges and induce the correct (square root) singularity at the leading edges. Work is presently under way by the author to determine the expansion coefficients $a_{m}^{(n)}$.

4. Low-solidity expansions. Recent renewed interest in propeller-driven aircraft has resulted in interesting "propfan" designs, which may be expected to behave aeroelastically more like turbomachinery fan rotors than propellers. For low-solidity cascades representative of propellers or propfans, it seems reasonable to seek asymptotic expansions in terms of a blade spacing parameter. When the blade spacing is large, the first term in such an expansion is the isolated airfoil solution, and the second term represents a correction arising from the cascade effect. A suitable limit process in this case is

$$
\bar{s}_{2}=\lambda \rightarrow \infty, \quad \bar{s}_{1} / \bar{s}_{2} \text { fixed }(\theta \text { fixed }),
$$

which preserves the stagger.

Supersonic Flow. The reduced cascade kernel $G$ can be written as a product of the isolated airfoil kernel $G_{I}$ and a "cascade function" $C$;

$$
G(\tau)=G_{I}(\tau) \cdot C(\tau)
$$

where

$$
G_{I}(\tau)=\frac{-i \sqrt{\tau^{2}-\bar{k}^{2}}}{\tau-\bar{k} / M}
$$

and

$$
\begin{aligned}
C(\tau) & =\sum_{m=-\infty}^{\infty} \exp \left\{i m\left(\Omega-\bar{s}_{1} \tau\right)-i|m| \bar{s}_{2} \beta \sqrt{\tau^{2}-\bar{k}^{2}}\right\} \\
& =i \sin \left(\bar{s}_{2} \beta \sqrt{\tau^{2}-\bar{k}^{2}}\right) /\left[\cos \left(\bar{s}_{2} \beta \sqrt{\tau^{2}-\bar{k}^{2}}\right)-\cos \left(\Omega-\bar{s}_{1} \tau\right)\right]
\end{aligned}
$$

For an isolated blade, $C(\tau) \equiv 1$, corresponding to $m=0$ in the infinite sum in Eq. (27). If we write

$$
\Delta C(\tau)=C(\tau)-1=\sum_{m=1}^{\infty} 2 \cos \left[m\left(\Omega-\bar{s}_{1} \tau\right)\right] \exp \left\{-i m \bar{s}_{2} \beta \sqrt{\tau^{2}-\bar{k}^{2}}\right\},
$$

then the governing equation for the upwash, Eq. (1), can be written as

$$
\bar{v}_{0}(\bar{x})=\frac{i \beta}{2} \int_{-\infty}^{\infty} G_{I}(\tau+\bar{k} M) F_{0}(\tau+\bar{k} M) e^{i \bar{x} \tau} d \tau+\Delta \bar{v}_{0}(\bar{x}),
$$


with

$$
\Delta \bar{v}_{0}(\bar{x})=\frac{i \beta}{2} \int_{-\infty}^{\infty} G_{I}(\tau+\bar{k} M) F_{0}(\tau+\bar{k} M) \Delta C(\tau+\bar{k} M) e^{i \bar{x} \tau} d \tau
$$

representing the cascade effect on the upwash on the reference blade. If we treat $\Delta \bar{v}_{0}(\bar{x})$ as known, Eq. (29) supplemented with Eq. (2) can be solved [12] to yield

$$
\begin{gathered}
F_{0}(\tau+\bar{k} M)=\sum_{n=0}^{\infty} c_{n} \int_{-\infty}^{\infty} \frac{(u+k)}{\sqrt{(u+\bar{k} M)^{2}-\bar{k}^{2}}} j_{n}(u) j_{0}(\tau-u) d u \\
c_{n}=\frac{(2 n+1)}{\pi \beta i^{n}} \int_{-1}^{1}\left[\bar{v}_{0}(\bar{x})-\Delta \bar{v}_{0}(\bar{x})\right] P_{n}(\bar{x}) d \bar{x}
\end{gathered}
$$

where $j_{n}, P_{n}$ are spherical Bessel functions and Legendre polynomials, respectively.

Proceeding as in the low-frequency case, we assume an expansion of the form of Eq. (6) with $\varepsilon=1 / \lambda \rightarrow 0$. From the Riemann-Lebesgue lemma it follows that $\Delta \bar{v}_{0}(\bar{x}) \rightarrow 0$ on the airfoil $(|\bar{x}|<1)$, and the leading term in (6) represents the isolated airfoil solution, given by Eqs. $(31,32)$ on setting $\Delta \bar{v}_{0}(\bar{x}) \equiv 0$. The succeeding terms in the expansion can then be found by first obtaining an asymptotic expansion for the integral (30),

$$
\Delta \bar{v}_{0}(\bar{x})=\nu_{1}(\varepsilon) \Delta \bar{v}_{0}^{(1)}(\bar{x})+\nu_{2}(\varepsilon) \Delta \bar{v}_{0}^{(2)}(\bar{x})+\cdots,
$$

and then solving a sequence of isolated airfoil problems for the terms of order $\nu_{1}, \nu_{2} \cdots$. The method of steepest descent, or a combination of the method of stationary phase and Laplace's method, can be used to evaluate the integral in (30) for $\lambda \rightarrow \infty$. Evidently $\nu_{1}=1 / \sqrt{\lambda}$, from the contribution of the stationary points of the exponents of $\Delta C(\tau)$.

Subsonic Flow. The above procedure is readily extended to subsonic cascades. By factoring out the isolated airfoil kernel, as in Eq. (25), a set of relations analogous to Eqs. $(29,30)$ is obtained. Proceeding as before, the successive terms in the expansion for the solution can be obtained by solving a sequence of isolated airfoil problems. In the incompressible case these solutions can be expressed in closed form in terms of Bessel functions $J_{n}$. In this case the upwash correction term $\Delta \bar{v}_{0}$ involves only Laplace-type integrals, and the first cascade correction term is expected to be of order $1 / \lambda$ as $\lambda \rightarrow \infty$.

5. Numerical results and discussion. Typical results calculated by the present asymptotic formulas are shown in Figs. 2-9, including comparisons with the full unsteady solution and with previously obtained approximations. In all of these calculations, the uniformly valid expansion coefficients were used. For convenience, lift and moment coefficients are introduced as follows:

$$
C_{F v}=-F_{0}(\bar{k} M) / \bar{v}_{0_{b}}, \quad C_{M \alpha}=i F_{0}^{\prime}(\bar{k} M) / 2 \alpha_{0} .
$$

Data is presented for a low-solidity cascade with no Mach wave reflections, for which $F_{0}(\bar{k} M)=a_{0}$ and $F_{0}^{\prime}(\bar{k} M)=a_{1} / 3$, and for Verdon's cascade A, for which the corresponding expressions are given in [4]. 
Figures 2 and 3 compare the present approximation of lift and moment coefficients $C_{F v}$ and $C_{M \alpha}$ with previously obtained (nonuniform) solutions [1,2] and the full unsteady solution [8], for Verdon's cascade A at progressively higher reduced frequencies. The present uniform approximation is seen to be remarkably accurate, even for the higher reduced frequencies. The standard nonuniform approximation is also quite good, except in the vicinity of $\sigma=0$ or $360^{\circ}$. Also, the latter approximation is restricted to the subresonant region.

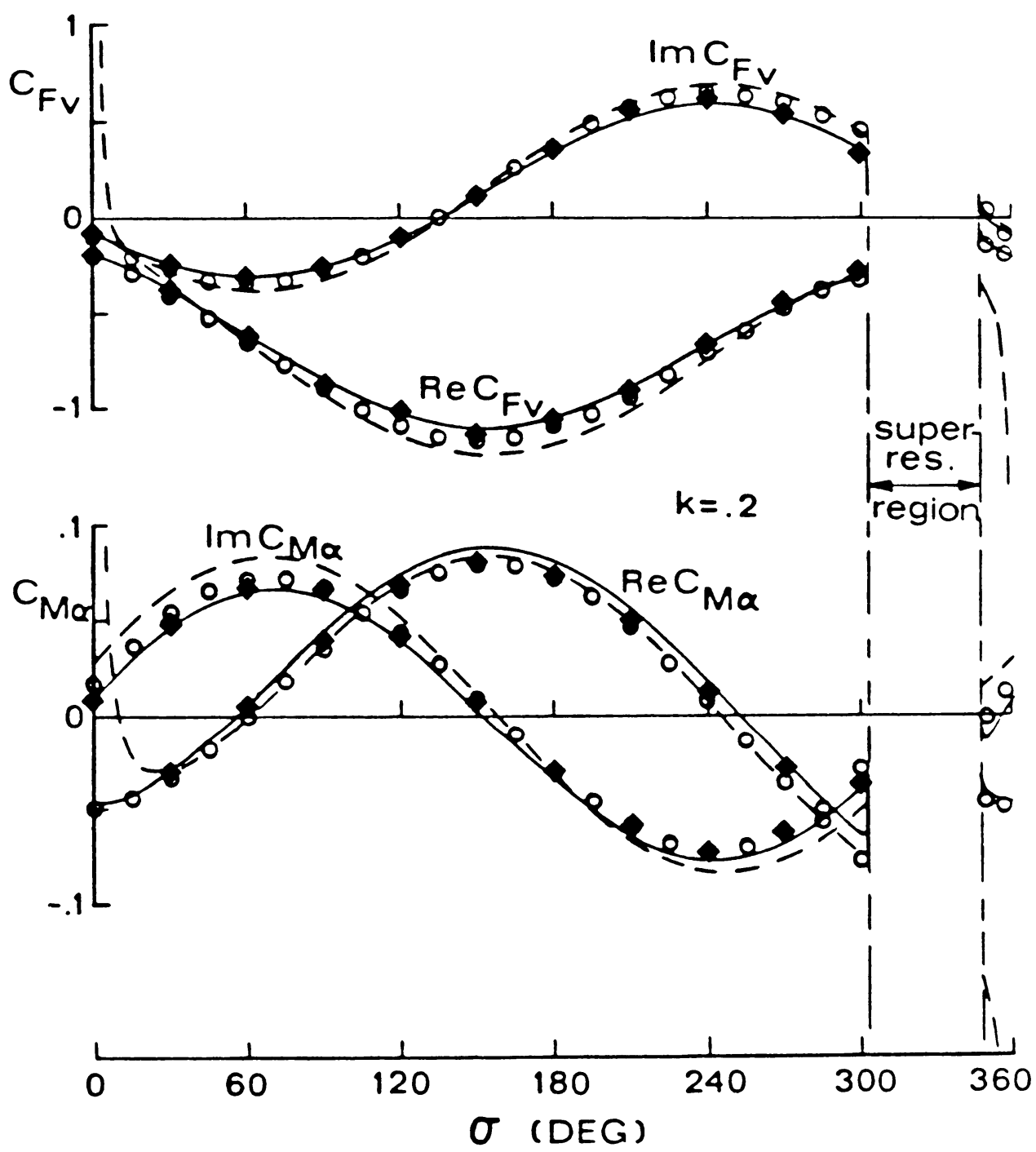

FIG. 2. Comparison of low-frequency approximations to lift and moment coefficients for Verdon's cascade A: (O) present approximations without $T$-term; $(\diamond)$ present approximations with $T$-term; (- $)$ nonuniform approximations [1, 2]; (-) full unsteady solution [8]. $M=1.345, \theta=59.5^{\circ}, \bar{s}_{2}=0.8, a=0$. 
As cascade solidity is decreased, the accuracy of the asymptotic solutions at any fixed reduced frequency appears to decrease. This is illustrated in Figs. 4 and 5 for the low-solidity cascade with no Mach wave reflections. Note that for this cascade the nonuniform approximations break down surprisingly quickly with increasing reduced frequency. The region of nonuniformity now extends over a wider range of interblade
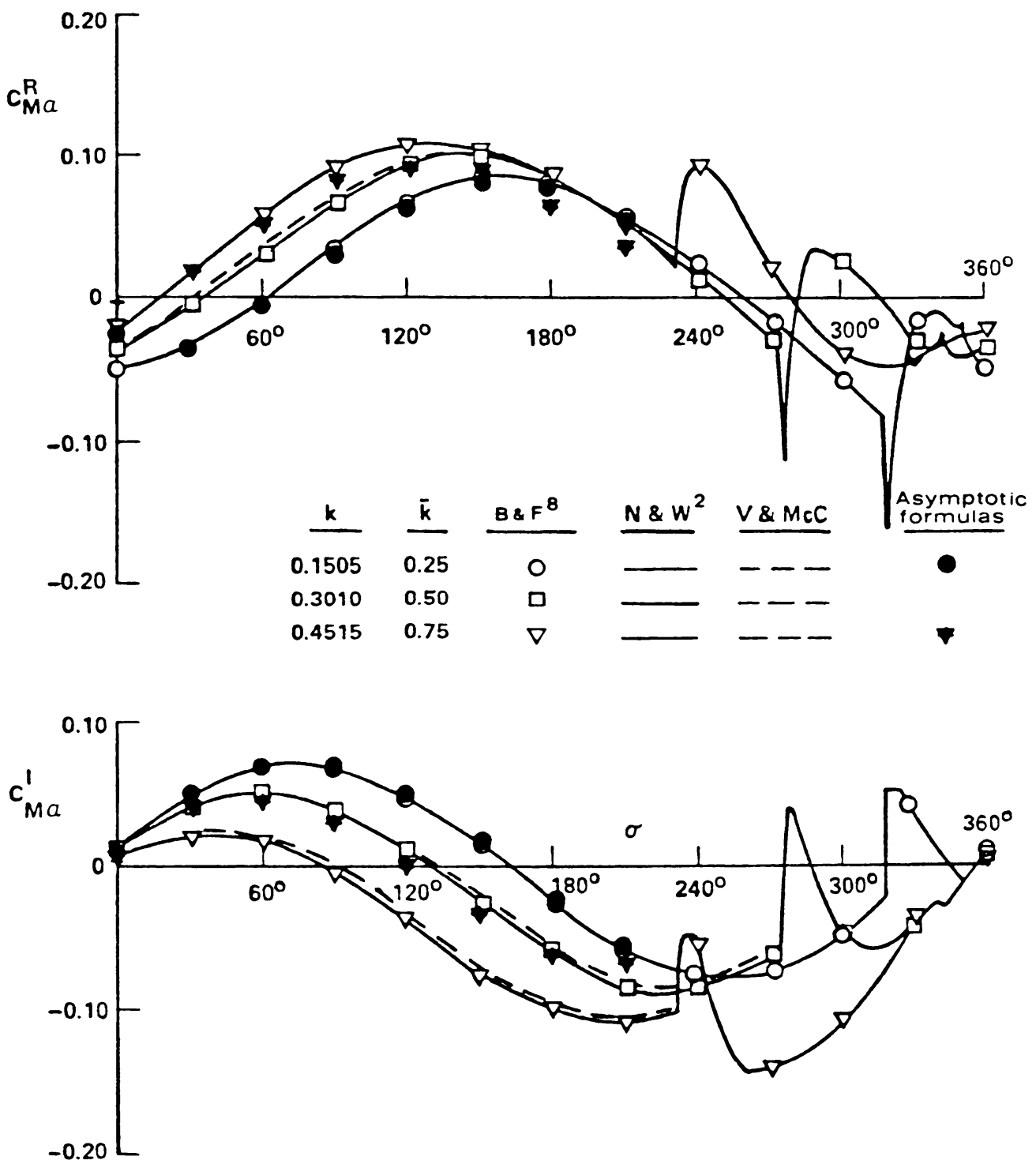

FIG. 3. Comparison of present low-frequency approximations to moment coefficient due to torsion for Verdon's cascade A. 


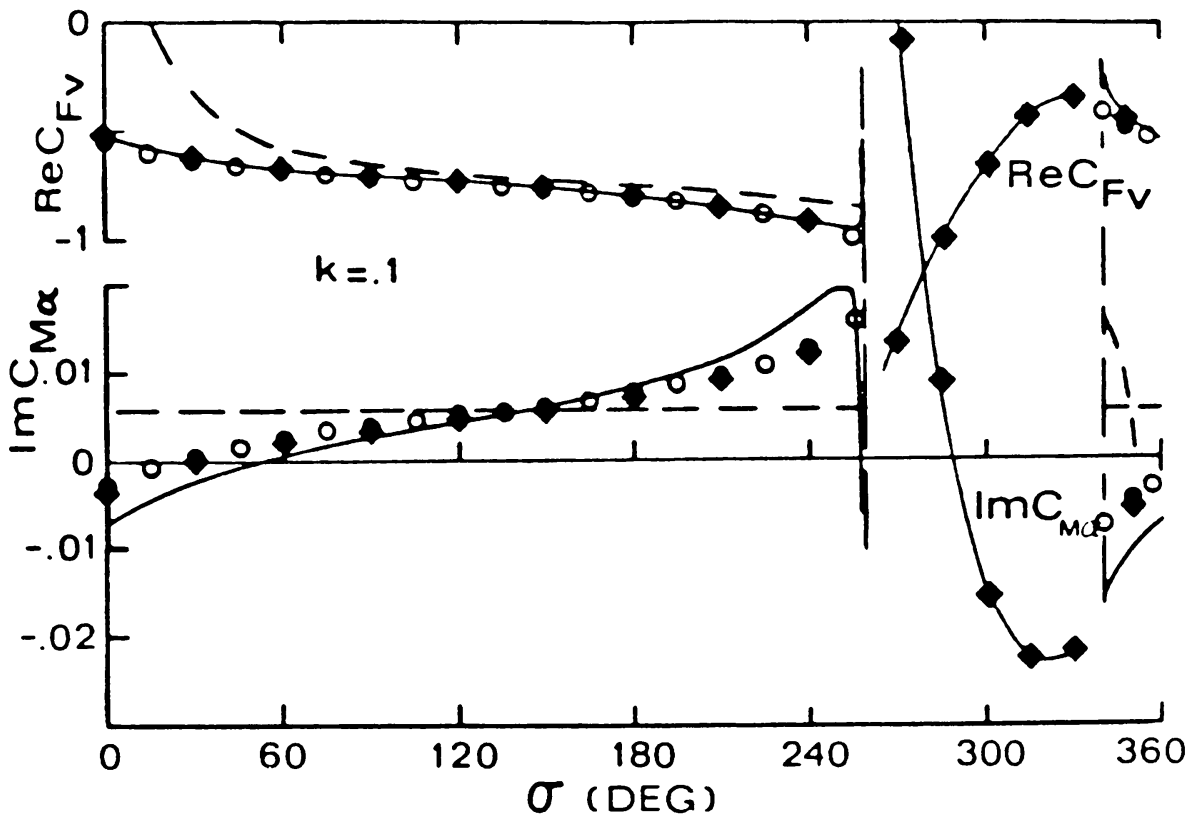

FIG. 4. Comparison of lift and moment coefficients for low-solidity cascade. Legend as in Fig. 2. $M=1.3$, $\theta=60^{\circ}, \bar{s}_{2}=2.5, a=0, k=0.1$.

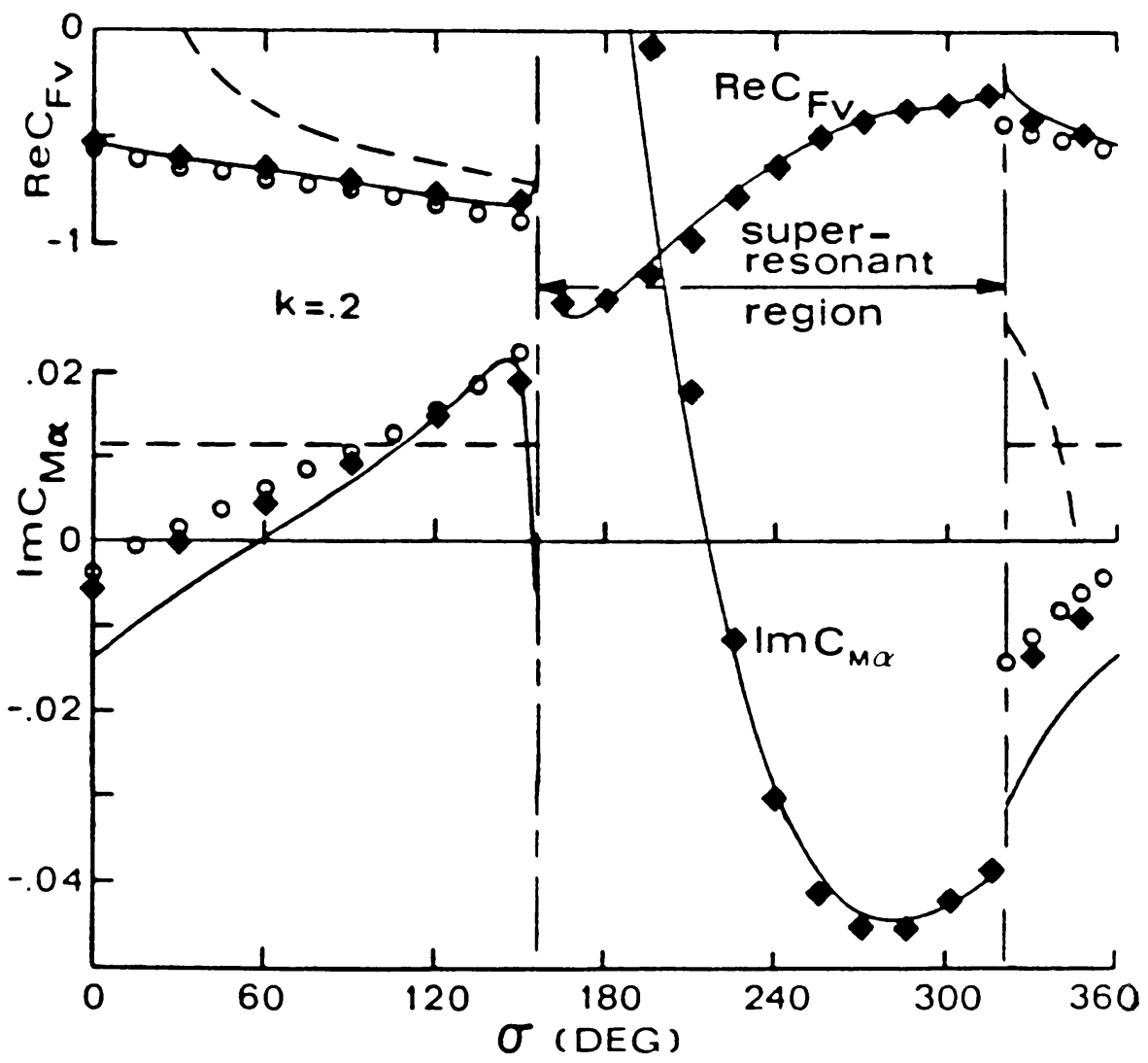

FIG. 5. Same as Fig. 4 at a reduced frequency $k=0.2$. 
phase angles, and the improvements obtained by using the present approximations are significant. Also note that the standard approximation predicts that $\operatorname{Im}\left\{C_{M_{\alpha}}\right\}$ is independent of interblade phase angle for these cascades, suggesting (incorrectly) that the stability margin for torsional oscillations should be independent of $\sigma$.

It should also be noted that the superresonant region increases as the solidity decreases, for fixed $k$. This is another indication that important cascade effects remain in the low-solidity limit and should not be ignored in propeller/propfan applications. The above observations suggest that it becomes more difficult to obtain useful engineering approximations as the blade spacing increases. This may appear paradoxical, since cascade effects due to the mutual interference of the infinite set of blades and vortex wakes should become weaker as the blades move farther apart. While this is certainly true, one must remember that a first-order expansion in a frequency parameter imposes restrictions on the validity of the solution. These restrictions are more severe in the far field than close to the source of the disturbance. Thus it is not surprising that such an expansion should become more accurate as the blades move closer, since more of the blades which dominate the interference effect on the reference blade are now within the near field.

Figures 6-9 illustrate how the accuracy of the present approximations varies with reduced frequency. In Figs. 6 and 7 the asymptotic solution for $C_{F v}$ and $C_{M \alpha}$ is compared to the full unsteady solution for Verdon's A, for typical values of $k$ and $\sigma$ of interest in aeroelastic analyses. For this cascade the critical reduced frequency for single-degree-offreedom torsional flutter is about 0.57 for $a=0$, and the critical interblade phase angle is close to $30^{\circ}$. In the absence of structural damping, torsional flutter occurs when $\operatorname{Im}\left\{C_{M \alpha}\right\}$ $>0$. Figures 8 and 9 show similar plots for the low-solidity cascade.

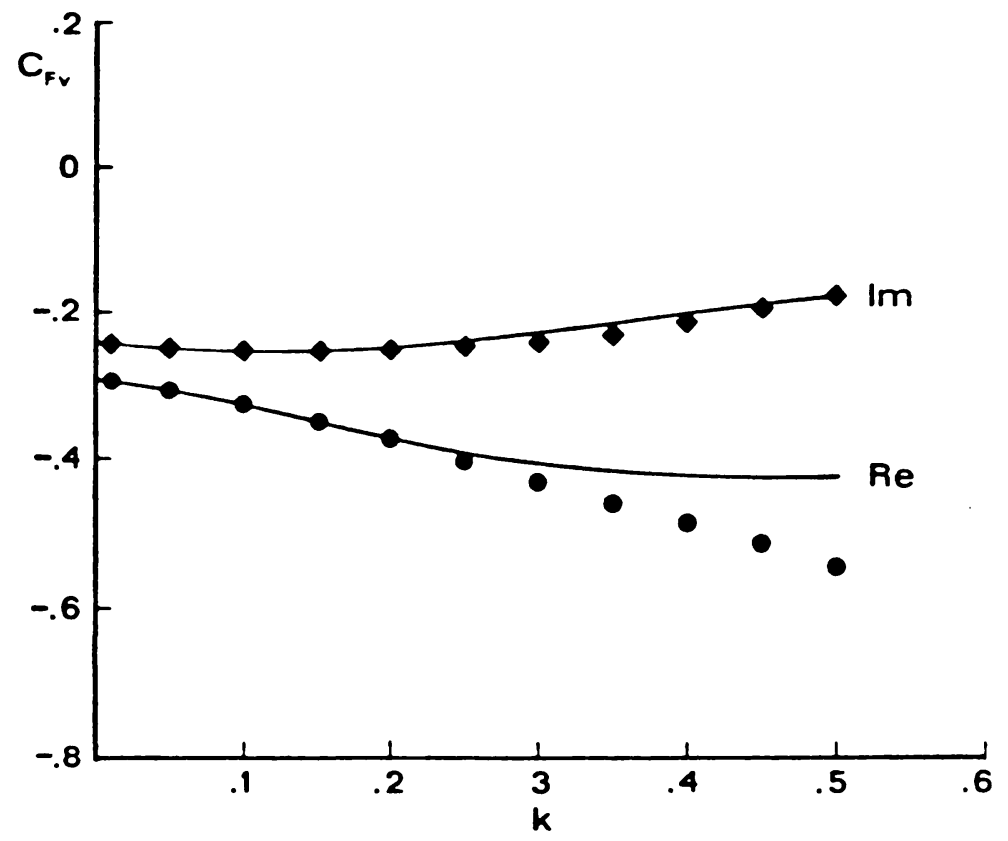

FIG. 6. Behavior of approximation of $C_{F v}$ as a function of reduced frequency $k:(\bullet, \diamond)$ asymptotic solution; (-) full unsteady solution. Verdon's cascade A, $a=0, \sigma=30^{\circ}$. 


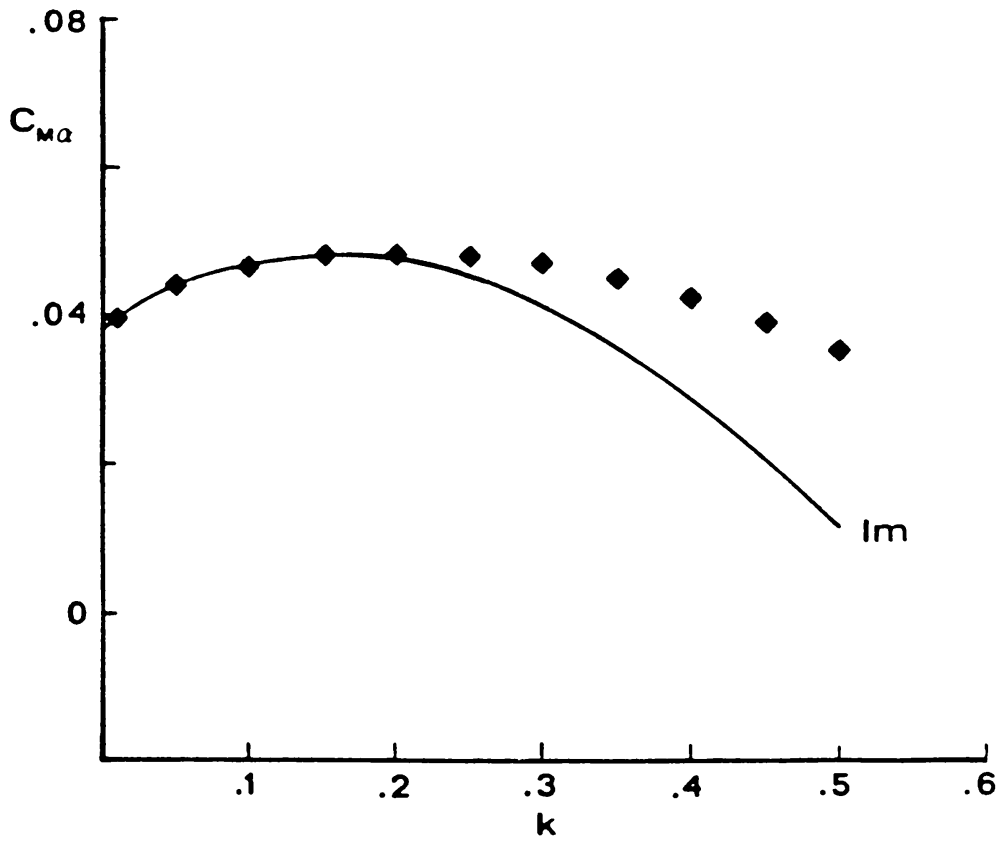

FIG. 7. Same as Fig. 6, for $\operatorname{Im} C_{M \alpha}$.

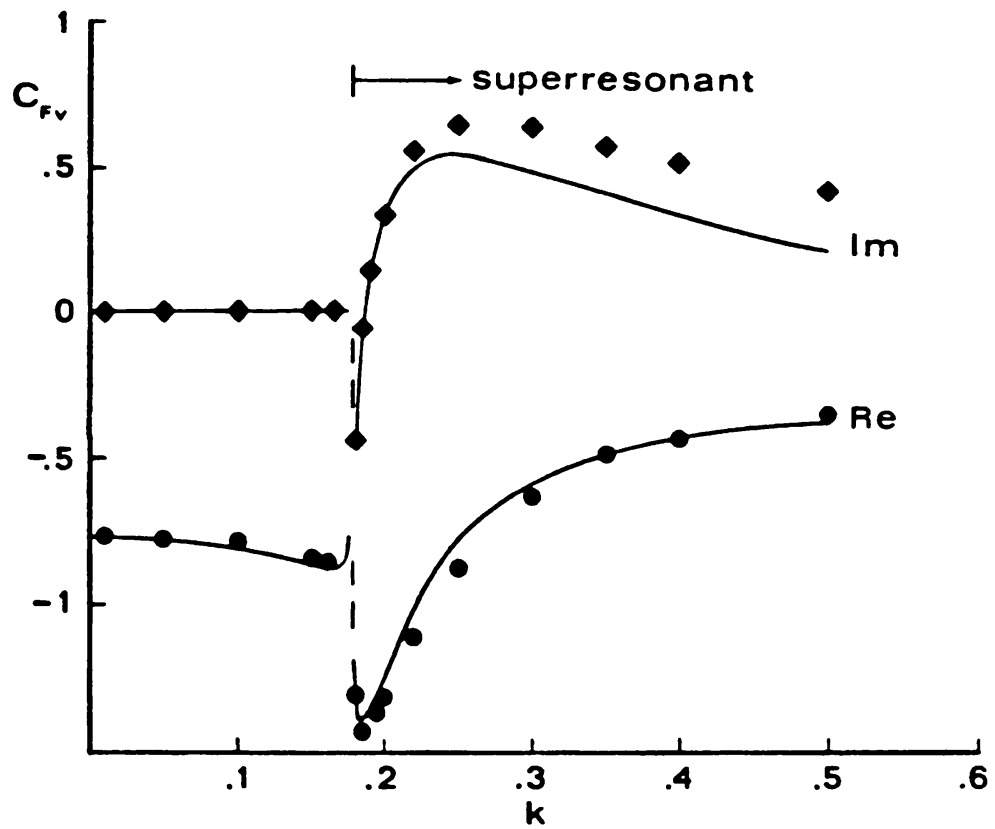

FIG. 8. Behavior of approximation of $C_{F v}$ as a function of reduced frequency for low-solidity cascade (Fig. 4), for $\sigma=180^{\circ}$. Legend as in Fig. 6 . 


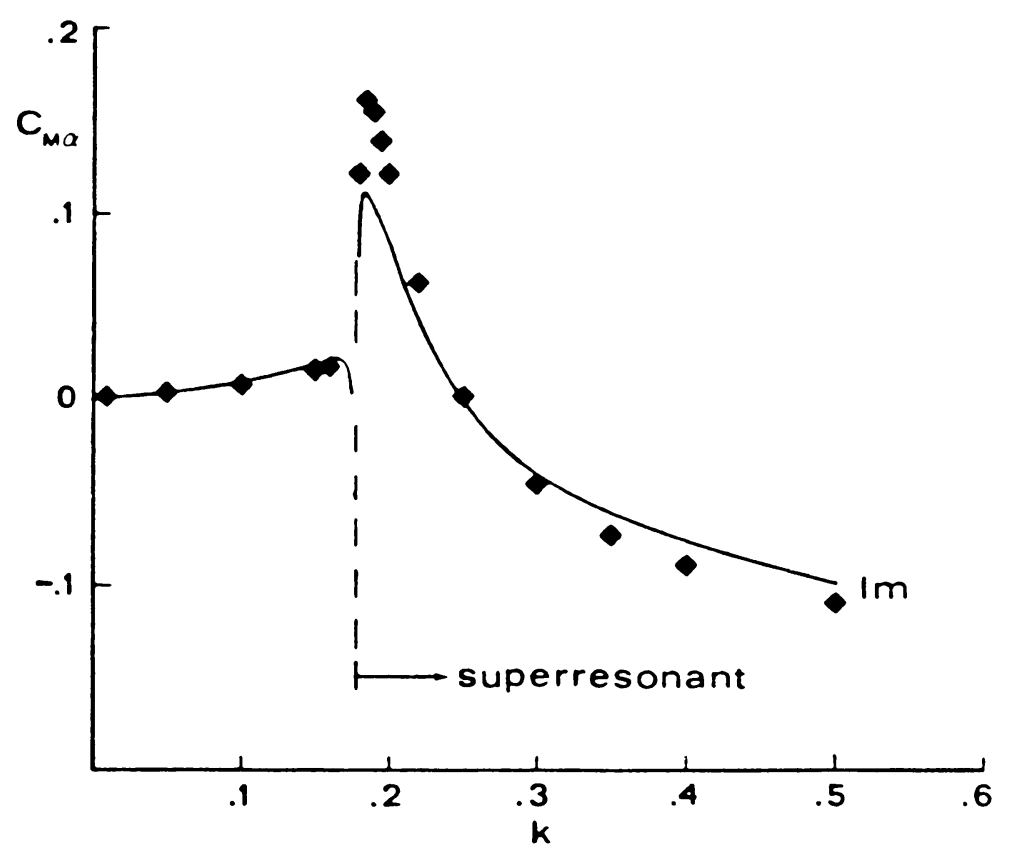

FIG. 9. Same as Fig. 8, for $\operatorname{Im} C_{M \alpha}$.

6. Conclusions. The main conclusions from this study can be summarized as follows:

1. Approximations which are sufficiently accurate to be useful in aeroelastic calculations can be obtained for both the subresonant and the superresonant operating regions by solving a sequence of quasi-static problems.

2. For cascades in subsonic axial flow, a nonuniformity with respect to interblade phase angle is to be expected. The location of the singularity can be moved by suitably redefining the limit process $k \rightarrow 0$, permitting uniformly valid expansions to be obtained separately for the subresonant and superresonant regimes.

3. For high-solidity cascades, the effect of Mach wave reflections produces a strong dependence on interblade phase angle in the lift and moment coefficients. The lowfrequency approximations are remarkably accurate, even for moderately high reduced frequencies.

4. The cascade effect remains surprisingly strong in the low-solidity limit and should therefore not be neglected in propeller/propfan applications. Low-solidity expansions can be obtained by solving a sequence of isolated airfoil problems.

\section{REFERENCES}

[1] M. Kurosaka, On the unsteady cascade with a subsonic leading edge-an exact first order theory: Parts 1 and 2, ASME J. Eng. Power 96, 13-31 (Jan. 1974)

[2] T. Nagashima and D. S. Whitehead, Linearized supersonic unsteady flow in cascades, Great Britain Aeronautical Research Council R \& M 3811 (1978)

[3] J. A. Strada, W. R. Chadwick and M. F. Platzer, Aeroelastic stability analysis of supersonic cascades, ASME Paper 78-GT-151 (1978) 
[4] O. O. Bendiksen and P. Friedmann, Coupled bending-torsion flutter in cascades with applications to fan and compressor blades, UCLA Rept. ENG-8072, Mechanics and Structures Dept. (1980)

[5] O. O. Bendiksen, A uniformly valid asymptotic solution for unsteady subresonant flow through supersonic cascades, AIAA J. 22, 154-157 (Jan. 1984)

[6] F. Lane and M. Friedman, Theoretical investigation of subsonic oscillatory blade-row aerodynamics, NACA TN-4136 (1958)

[7] D. S. Whitehead, Vibration and sound generation in a cascade of flat plates in subsonic flow, Great Britain A. R. C. R \& M 3685 (1970)

[8] O. O. Bendiksen and P. Friedmann, Bending-torsion flutter in supersonic cascades, AIAA J. 19, 774-781 (June 1981)

[9] I. N. Sneddon, Mixed boundary value problems in potential theory, North-Holland, Amsterdam, 1966

[10] J. Kevorkian and J. D. Cole, Perturbation methods in applied mathematics, Springer-Verlag, New York, 1981

[11] J. M. R. Graham and I. Kullar, Small perturbation expansions in unsteady aerofoil theory, J. Fluid Mech. 83, 209-224 (1977)

[12] O. O. Bendiksen, Low-frequency expansions for unsteady flow in a supersonic cascade, MAE Report, Princeton University (1984) 